\title{
Pronounced optical gain attained in Ag/AZO structure for solar cell applications
}

\section{Y. Y. Feng, H. P. Zhou*, Y. Xiang*, H. Ji, X. H. Chang, C. Wang}

School of Energy Science and Engineering, University of Electronic Science and Technology of China, 2006 Xiyuan Ave, West High-Tech Zone, Chengdu, Sichuan 611731, China

\begin{abstract}
Recently plasmonic effect has attracted tremendous interests due to its ability of light trapping and improving the photovoltaic performances of solar cells. In this letter, we propose a solution method based fabrication route of $\mathrm{Ag}$ plasmons and realize a pronounced optical gain in $\mathrm{ZnO}: \mathrm{Al}(\mathrm{AZO})$ thin film, which is strongly beneficial to harvest incident sunlight in thin film solar cells. Processing parameters, such as spinning speed, coating times and post-annealing temperature, could be regulated to optimize the plasmon effects of silver particles. The reflectance of silver particles coated AZO (Ag/AZO) is reduced by $\sim 18 \%$, and the transmittance is enhanced by $\sim 40 \%$ simultaneously. The underlying mechanisms are discussed in combination with the microstructures of the silver particles.
\end{abstract}

Key words: Particles, AZO, Reflectance, Transmittance, Plasmons, Deposition

\section{Introduction}

In the last several decades, nano noble metal materials, such as silver (Ag) and gold (Au), have attracted intensive attentions due to their wide applications in medical optical imaging [1], drugs [2] and solar cells [3] etc. Silver particles with a lower cost could be elaborately controlled to form surface plasmon polaritons (SPPs) with various shapes [4] and sizes [3] to trap light, which is the underlying mechanism for antireflection. As an example, Temple and Dligatch [5] proposed a structure of $\mathrm{Ag} / \mathrm{SiO}_{2} / \mathrm{Si}$ where the introduction of $\mathrm{Ag}$ layer with an optimized thickness of $5 \mathrm{~nm}$ was able to reduce

\footnotetext{
${ }^{*}$ Corresponding authors.

E-mail address: haipzhou@uestc.edu.cn (H. P. Zhou), xiang@uestc.edu.cn (Y. Xiang)
} 
the surface reflection by $\sim 10 \%$. On the other hand, $\mathrm{Ag}$ particles coated on the surface of AZO thin film can enhance the light scattering and lead to an increased transmittance of Ag/AZO [6]. For example, Theuring, et al. [7] presented a sandwich structure of $\mathrm{AZO} / \mathrm{Ag} / \mathrm{AZO}$ whose transmittance was increased from $\sim 70 \%$ to $\sim 80 \%$ due to the introduction of the intermediate $\mathrm{Ag}$ layer. That is to say, the reflection (transmission) of window layers can be effectively minimized (enhanced) by applying Ag SPPs. However, it seems to be difficult to attain a pronounced optical gain via decreasing the reflection and simultaneously increasing the transmittance in the window layer, to harvest more incident sunlight $[5,7$, 8] by using metal SPPs.

As for the fabrication of silver plasmons, physical methods, such as magnetron sputtering [9] and electron beam evaporation [5] are commonly used. However, these physical methods are practically limited by a high cost and a complicated processing procedure. Recently we have developed an easy chemical reduction method $[10,11]$ to make the fabrication process to be easier, safer and more controllable. The morphologies and microstructures of the silver particles can be effectively tuned by the processing parameters. It was found that both the as-fabricated and thermally annealed silver particles significantly enhance the light transmission, and simultaneously reduce the reflectance on the AZO thin film surface due to the SPPs effect.

\section{Experimental details}

Silver nitrate $\left(\mathrm{AgNO}_{3}\right)$ was used as a raw material to prepare silver particles. Citric acid three sodium $\left(\mathrm{Na}_{3} \mathrm{C}_{6} \mathrm{H}_{5} \mathrm{O}_{7} \cdot 2 \mathrm{H}_{2} \mathrm{O}\right)$, polyvinyl pyrrolidone (PVP) and deionized (DI) water were used as reductant, stabilizer and solvent, respectively. The substrate was $350 \mathrm{~nm}$ thick AZO thin film magnetron sputtering grown on glass substrate. The mixed solution of $18 \mathrm{~mL}$ DI water, $0.8 \mathrm{~g}$ PVP, 0.5 $\mathrm{g} \mathrm{Na}_{3} \mathrm{C}_{6} \mathrm{H}_{5} \mathrm{O}_{7} \cdot 2 \mathrm{H}_{2} \mathrm{O}$ and $0.08 \mathrm{~g} \mathrm{AgNO}{ }_{3}$ was stirred by a magnetic stirring apparatus to form a uniform 
precursor solution, and then introduced into a sealed reaction kettle to avoid the atmosphere exposure. The reaction kettle was put inside a thermostat set at the temperature of $180{ }^{\circ} \mathrm{C}$ for $20 \mathrm{~h}$ for the formation of silver colloid. Silver colloid was subsequently deposited on the AZO substrate via a spin coater to form the $\mathrm{Ag} / \mathrm{AZO}$ structure. Finally, as-fabricated $\mathrm{Ag} / \mathrm{AZO}$ was thermally annealed in an annealing equipment as schematically shown in Fig. 1.

Light reflectance and transmittance spectra of $\mathrm{Ag} / \mathrm{AZO}$ were measured by using Agilent UV/VIS/NIR spectrophotometer (Cary 5000). Scanning electron microscopy (SEM) measurements of $\mathrm{Ag} / \mathrm{AZO}$ were taken on FEI Inspect $\mathrm{F}$ working at $20 \mathrm{kV}$. X-Ray diffraction (XRD) measurements were performed on Bede D1 whose working voltage, operating current and X-ray wavelength were set at 50 $\mathrm{kV}, 35 \mathrm{~mA}$ and $0.15402 \mathrm{~nm}$, respectively.

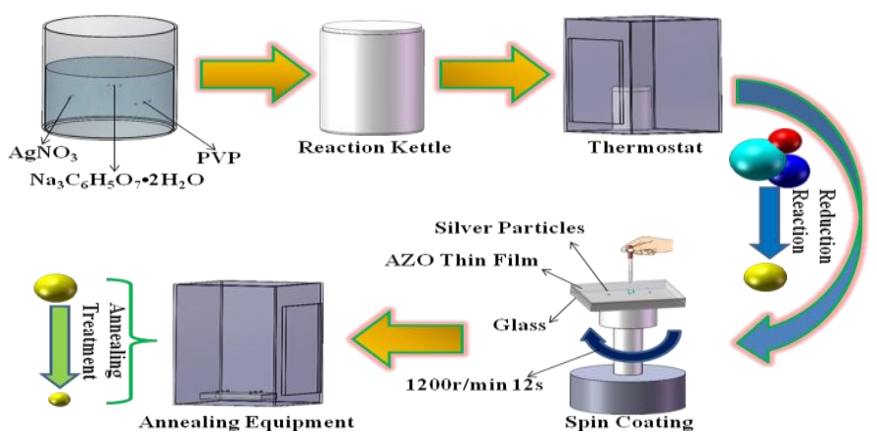

Fig. 1. The schematic fabrication flow of the silver particles

\section{Result and discussion}

Fig. 2(a-d) show the XRD spectra of the bare and 17 times silver colloid coated AZO thin films: bare AZO without silver colloid coating (Ag-0/AZO) (a), coated AZO without post-annealing (Ag-17/AZO) (b), coated AZO with a 4-hour post-annealing at $150{ }^{\circ} \mathrm{C}(\mathrm{Ag}-17 / \mathrm{AZO} /$ annealing with 150 $\left.{ }^{\circ} \mathrm{C}\right)(\mathrm{c})$, and coated AZO with a 4-hour post-annealing at $200{ }^{\circ} \mathrm{C}\left(\mathrm{Ag}-17 / \mathrm{AZO} /\right.$ annealing with $\left.200{ }^{\circ} \mathrm{C}\right)$ (d). Apart from the peak located at $\sim 34.3^{\circ}$ which is definitely recognized as the $\mathrm{ZnO}(002)$ plane shown in Fig. 2(a), another peak located at $2 \theta=62.540^{\circ}$ attributed to the $\operatorname{AgO}(-221)$ plane appears. The 
occurrence of $\mathrm{AgO}$ should be ascribed to the oxidation of $\mathrm{Ag}$ in air. In Fig. 2(c) there are three diffraction peaks, apart from $\mathrm{ZnO}(002)$ and $\mathrm{AgO}(-221)$, another standard (111) plane of $\mathrm{Ag}$ is verified as $2 \theta=38.120^{\circ}$. The appearance of $\mathrm{Ag}$ associated diffraction peak indicates that the $150{ }^{\circ} \mathrm{C}$ annealing makes Ag particle crystallize. As shown in Fig. 2(d), the diffraction peak of Ag disappears, which means that the annealing temperature of $150{ }^{\circ} \mathrm{C}$ is more beneficial to crystallization of $\mathrm{Ag}$ particles than $200{ }^{\circ} \mathrm{C}$.
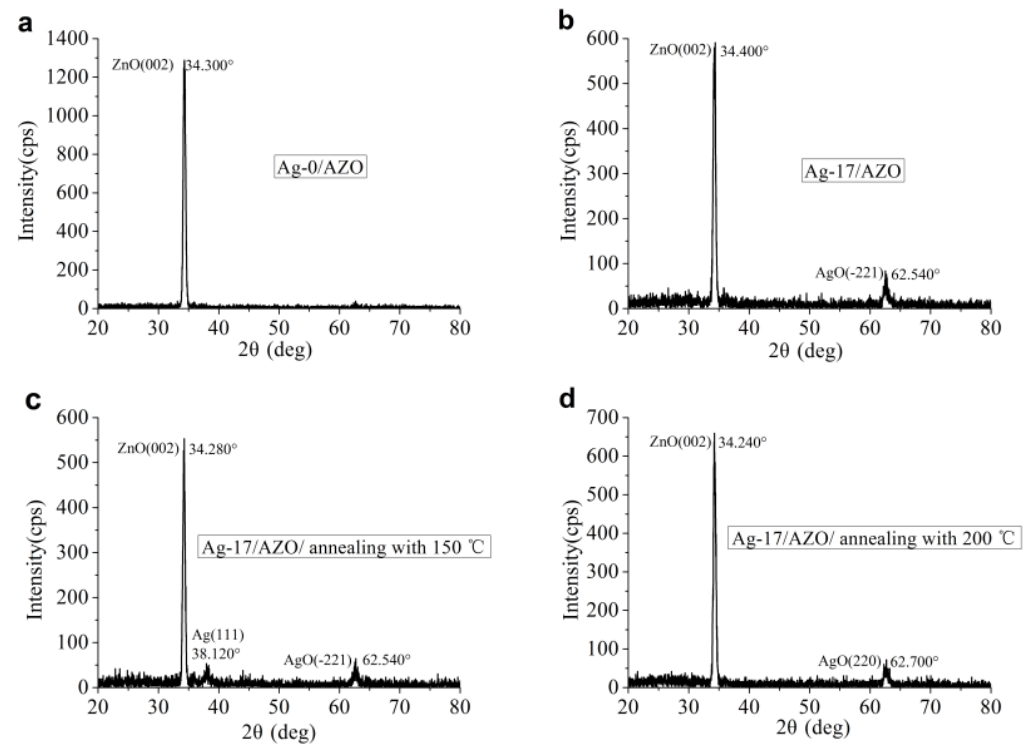

Fig. 2. XRD spectra of the bare and Ag particles coated AZO thin films (see text)

The surface morphologies of Ag/AZO were examined by SEM. Fig. 3(a) [(b)] shows the top-view image of Ag-0/AZO [Ag-17/AZO]. A typical surface morphology of crystal AZO is represented in Fig. 3(a). There are sparse Ag or AgO particles situated on the surface of Ag-17/AZO as shown in Fig. 3(b). Interestingly, the particles are irregular and separated with various shapes of hexagon and square (outlined in the figure by dashed lines). The underling mechanism is out of the scope of this letter and will be studied elsewhere. The morphology of Ag-17/AZO/annealing with 150 ${ }^{\circ} \mathrm{C}$ is shown in Fig. 3(c), where the particle shape becomes sphere with a markedly reduced size. These observations mean that the $150{ }^{\circ} \mathrm{C}$ post-annealing can lead to a discoalescence of the bigger silver 
particles into chemically reshaped smaller particles. With an increasing annealing- temperature, the dewetting speed of silver colloid is accelerated to result in smaller particles [12]. Fig. 3(d) shows the SEM image of Ag-17/AZO/annealing with $200{ }^{\circ} \mathrm{C}$, where the particle size is further reduced and the distribution is more homogeneous. It is concluded that the post-annealing treatment could lead to the continuous decomposition and make the particles to be more homogeneous [3].
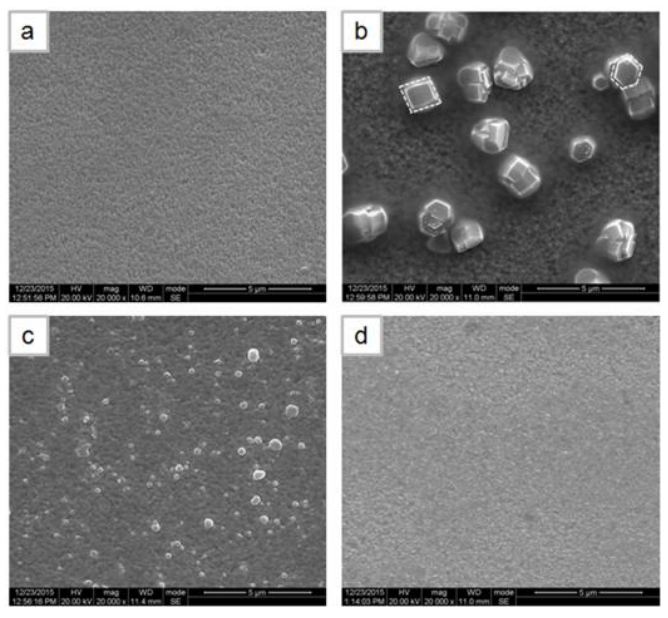

Fig. 3. Top-view SEM images of Ag-0/AZO (a), Ag-17/AZO (b), Ag-17/AZO/annealing with $150{ }^{\circ} \mathrm{C}$ (c), and Ag-17/AZO/ annealing with $200{ }^{\circ} \mathrm{C}(\mathrm{d})$.

The effects of the surface particles on the optical properties of AZO were also investigated. The reflectance spectra of the AZO thin films with different coating times of silver colloid are shown in Fig. 4(a), in which the reflectance of AZO coated with silver colloid is lower than that of bare AZO in a wide wavelength range covering the UV-VIS-NIR span. Silver colloid coated on the surface of AZO thin film could form SPPs where the collective oscillations of Ag particles' free electrons after light incidence lead to surface plasmon resonances (SPRs) and hence result in light trapping [13]. Meanwhile, as shown in Fig. 4(b), the transmittance of the Ag particles coated AZO drastically increases, which can be also ascribed to SPRs. Ag particles located at the interface between two $\underline{\text { mediums (air and AZO thin film) with different refractive indices scatter the light predominately into }}$ 
the higher one [6] as shown in Fig. 4(c). The reflectance and transmittance of Ag-17/AZO decreases and increases $\sim 10 \%$ and $\sim 25 \%$ respectively with respect to that of the bare AZO.

Annealing treatment is another significant factor influencing the optical properties of Ag/AZO.

As revealed in prior SEM measurements, post-annealing significantly reduces the silver particle size. The smaller silver particle is inclined to form quadrupolar mode leading to a decrease in the reflectance due to forward-scattering as its size decreases [3]. Meanwhile, the extinction which is simply the inverse of the transmittance increases with increasing sizes of silver particle [3], therefore the transmittance of Ag/AZO increases with decreasing silver particle sizes. The reflectance and transmittance of Ag-17/AZO/annealing with $150{ }^{\circ} \mathrm{C}$ decreases and increases about $15 \%$ and $33 \%$ respectively in comparison to $\mathrm{Ag}-0 / \mathrm{AZO}$. When the annealing temperature reaches $200{ }^{\circ} \mathrm{C}$, the silver particle is the smallest as shown in Fig. 3(d). Consequently, the reflectance and transmittance of Ag-17/AZO/ annealing with $200{ }^{\circ} \mathrm{C}$ decreases and increases by about $18 \%$ and $40 \%$ respectively in comparison to $\mathrm{Ag}-0 / \mathrm{AZO}$. In principle, there are two types of modes in the formed plasmon on the surface of AZO thin film, namely the SPPs mode and local surface plasmons (LSPs) mode. According to Fig. 4(a) and (b), the light absorption in the Ag silver colloid coated AZO is very low because of the high sum values of the reflectance and transmittance, and thus the effect of LSPs which tends to enhance absorption [14] is much weaker than that of SPPs in the present experiments. Furthermore, the occurrence of LSPs usually needs a small particle size $(1-100 \mathrm{~nm})$, and LSPs are prone to be transformed into SPPs when the particle size is increased [15]. As shown in Fig. 3(b-d), the size of Ag particle is much more than $100 \mathrm{~nm}$, and thus a dominant role of SPPs should be expected in our case. 

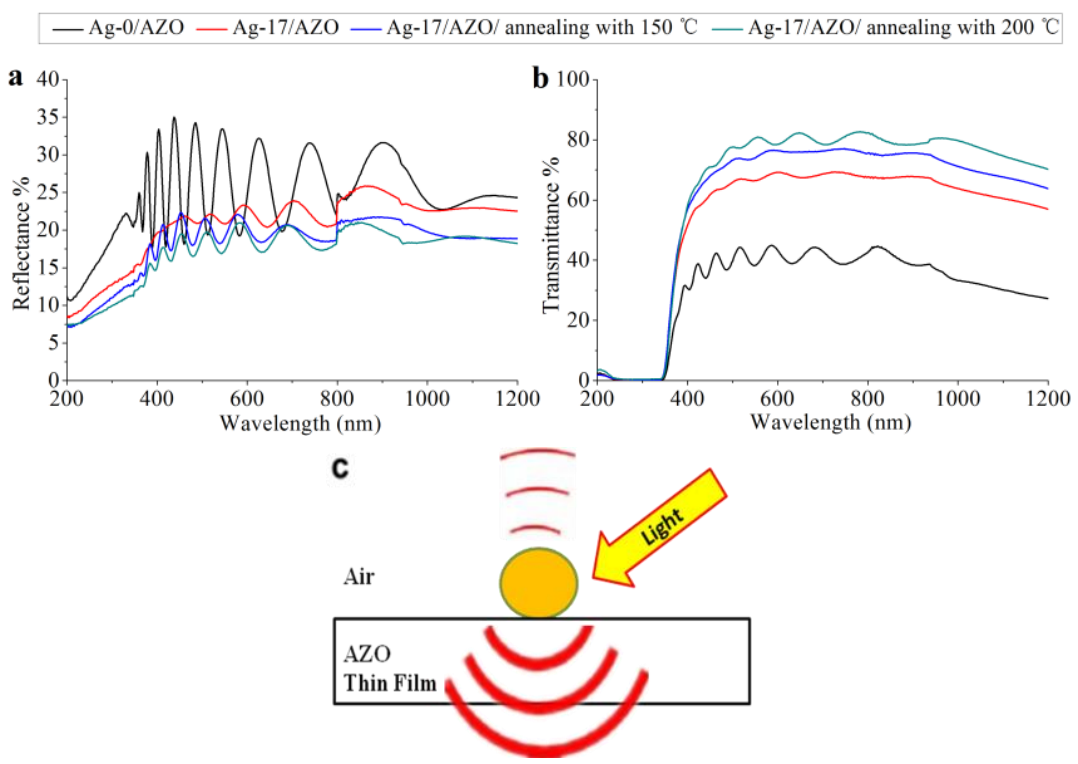

Fig. 4. The reflectance (a) and transmittance (b) spectra of the various samples. Ag particles scatters incident light into AZO thin film as shown in (c).

\section{Conclusion}

A pronounced optical gain is attained by a solution route based Ag SPPs on AZO substrate. The introduction of Ag SPPs with an appropriate post-annealing, which effectively tunes the microstructures and distribution of $\mathrm{Ag}$ particles, leads to a pronounced optical gain in the $\mathrm{Ag} / \mathrm{AZO}$ structure: a 18\% decrease in the reflectance and simultaneously a $40 \%$ increase in the transmittance.

The present Ag/AZO structure shows its huge prospect in improving the photovoltaic performance of thin film solar cells due to the pronounced optical gain.

\section{Acknowledgments}

This work was supported by National Science Foundation of China (Grant No. 51302028).

\section{References}

[1] J. Chen, F. Saeki, B.J. Wiley, H. Cang, M.J. Cobb, Z.-Y. Li, L. Au, H. Zhang, M.B. Kimmey, X. $\mathrm{Li}$, Gold nanocages: bioconjugation and their potential use as optical imaging contrast agents, Nano Lett 5 (2005) 473-477.

[2] D. Nayak, S. Ashe, P.R. Rauta, M. Kumari, B. Nayak, Bark extract mediated green synthesis of silver nanoparticles: Evaluation of antimicrobial activity and antiproliferative response against osteosarcoma, Mat Sci Eng C-Mater 58 (2016) 44-52.

[3] T. Temple, G. Mahanama, H. Reehal, D. Bagnall, Influence of localized surface plasmon excitation 
in silver nanoparticles on the performance of silicon solar cells, Sol Energ Mat Sol C 93 (2009) 1978-1985.

[4] B.J. Wiley, S.H. Im, Z.-Y. Li, J. McLellan, A. Siekkinen, Y. Xia, Maneuvering the surface plasmon resonance of silver nanostructures through shape-controlled synthesis, The Journal of Physical Chemistry B 110 (2006) 15666-15675.

[5] T.L. Temple, S. Dligatch, Role of the spacer layer in plasmonic antireflection coatings comprised of gold or silver nanoparticles, Journal of Photonics for Energy 5 (2015) 053095.

[6] S. Varlamov, Z. Ouyang, X. Zhao, D.S. Jung, Surface plasmon enhanced light-trapping in polycrystalline silicon thin-film solar, Photonics Global Conference, 2010, pp. 1-6.

[7] M. Theuring, M. Vehse, K. von Maydell, C. Agert, AZO-Ag-AZO transparent electrode for amorphous silicon solar cells, Thin Solid Films 558 (2014) 294-297.

[8] E. Placzek-Popko, K. Gwozdz, Z. Gumienny, E. Zielony, R. Pietruszka, B. Witkowski, S. Gieraltowska, M. Godlewski, W. Jacak, L.-B. Chang, Si/ZnO nanorods/Ag/AZO structures as promising photovoltaic plasmonic cells, J Appl Phys 117 (2015) 193101.

[9] B. Herrera, T. Bruna, D. Guerra, N. Yutronic, M. J. Kogan, P. Jara, Silver nanoparticles produced by magnetron sputtering and selective nanodecoration onto $\alpha$-cyclodextrin/carboxylic acid inclusion compounds crystals, Advances in Nanoparticles 2 (2013) 112-119.

[10] Y. Wang, Y. Zheng, C.Z. Huang, Y. Xia, Synthesis of Ag nanocubes 18-32 nm in edge length: the effects of polyol on reduction kinetics, size control, and reproducibility, Journal of the American Chemical Society 135 (2013) 1941-51.

[11] Q. Zhang, N. Li, J. Goebl, Z. Lu, Y. Yin, A systematic study of the synthesis of silver nanoplates: is citrate a "magic" reagent?, Journal of the American Chemical Society 133 (2011) 18931-9.

[12] H. Nasser, E. Özkol, A. Bek, R. Turan, High haze nature of textured Al:ZnO with Ag nanoparticles for light management in thin film solar cells, Optical Materials Express 5 (2015) 932.

[13] S.K. Sardana, V.S.N. Chava, V.K. Komarala, Morphology and optical properties of sputter deposited silver nanoparticles on plain, textured and antireflection layer coated textured silicon, Appl Surf Sci 347 (2015) 651-656.

[14] X. Fan, J. Fan, X. Hu, E. Liu, L. Kang, C. Tang, Y. Ma, H. Wu, Y. Li, Preparation and characterization of $\mathrm{Ag}$ deposited and $\mathrm{Fe}$ doped $\mathrm{TiO} 2$ nanotube arrays for photocatalytic hydrogen production by water splitting, Ceram Int 40 (2014) 15907-15917.

[15] W.A. Murray, S. Astilean, W.L. Barnes, Transition from localized surface plasmon resonance to extended surface plasmon-polariton as metallic nanoparticles merge to form a periodic hole array, Physical Review B 69 (2004). 